\title{
Anticonvulsant Drugs, Brain Glutamate Dehydrogenase Activity and Oxygen Consumption
}

\author{
Lourdes A. Vega Rasgado, ${ }^{1}$ Guillermo Ceballos Reyes, ${ }^{2}$ and Fernando Vega-Díaz ${ }^{1}$ \\ ${ }^{1}$ Neurochemistry Laboratory, Department of Biochemistry, National School of Biological Sciences, National Polytechnic Institute, \\ Carpio Y Plan de Ayala S/No., Col. Casco de Santo Tomás, 11340 México, DF, Mexico \\ ${ }^{2}$ Superior School of Medicine, National Polytechnic Institute, Plan de San Luis y Díaz Mirón S/No., Col. Casco de Santo Tomás, \\ 11340 México, DF, Mexico
}

Correspondence should be addressed to Lourdes A. Vega Rasgado, lourdes_vega_rasgado@hotmail.com

Received 17 November 2011; Accepted 18 December 2011

Academic Editor: T. W. Stone

Copyright ( 2012 Lourdes A. Vega Rasgado et al. This is an open access article distributed under the Creative Commons Attribution License, which permits unrestricted use, distribution, and reproduction in any medium, provided the original work is properly cited.

\begin{abstract}
Glutamate dehydrogenase (GDH, E.C. 1.4.1.3.) is a key enzyme for the biosynthesis and modulation of glutamate (GLU) metabolism and an indirect $\gamma$-aminobutyric acid (GABA) source, here we studied the effect of anticonvulsants such as pyridoxal phosphate (PPAL), aminooxyacetic acid (AAOA), and hydroxylamine (OHAMINE) on GDH activity in mouse brain. Moreover, since GLU is a glucogenic molecule and anoxia is a primary cause of convulsions, we explore the effect of these drugs on oxygen consumption. Experiments were performed in vitro as well as in vivo for both oxidative deamination of GLU and reductive amination of $\alpha$-ketoglutarate $(\alpha \mathrm{K})$. Results in vitro showed that PPAL decreased oxidative deamination of GLU and oxygen consumption, whereas AAOA and OHAMINE inhibited GDH activity competitively and also inhibited oxygen consumption when $\alpha \mathrm{K}$ reductive amination was carried out. In contrast, results showed that in vivo, all anticonvulsants enhanced GLU utilization by GDH and also decreased oxygen consumption. Together, results suggest that GDH activity has repercussions on oxygen consumption, which may indicate that the enzyme activity is highly regulated by energy requirements for metabolic activity. Besides, GDH may participate in regulation of GLU and, indirectly GABA levels, hence in neuronal excitability, becoming a key enzyme in seizures mechanism.
\end{abstract}

\section{Introduction}

Seizures are considered to be the result of an unbalance between excitatory and inhibitory systems, and it is believed that anticonvulsants exert their actions through enhancement of inhibitory-mediated transmission, the reduction of excitatory-mediated transmission, or a combination of both [1].

Glutamate (GLU) is considered the main excitatory neurotransmitter in the central nervous system (SNC) in mammals, besides, it is the precursor for the biosynthesis of gamma-aminobutyric acid (GABA), the main inhibitory neurotransmitter. It has been proposed that GLU may be involved in the pathogenesis of seizures [2], since many pathological processes in the SNC leading to the development of convulsive syndrome can increase the activity of glutamatergic transmission as the common end pathway [37].

Glutamate dehydrogenase (GDH, EC 1.4.1.3.) catalyzes the reversible deamination of $\mathrm{L}$-glutamate to 2-oxoglutarate. Evidences of the particular importance of GDH activity in the nervous system are GDH isolated from patients with heterogeneous neurological disorders, characterized by multisystem atrophy, displayed a marked reduction on its activity [8], with a similar trend in cortex and hippocampus of patients with temporal lobe epilepsy [9]. Increased activity of GDH has been reported in epileptic focus of human brain [10]. In addition, it has been proposed that pathogenesis of epilepsy in patients with hyperinsulinism/hyperammonemia syndrome may be related to an increasing GDH activity [11].

On the other hand, hypoxia is considered as a primary cause of seizures. Although this effect could be mediated 
by free radicals [12-15], many studies have shown that following hypoxic and ischemic injury in the brain, there is an excessive release of GLU and overstimulation of its receptors [16-18]. GDH might participate in this effect generating GLU, but also as a source of energy for the oxidative metabolism in the brain through the Krebs cycle.

The management of seizures in epileptic patients relies heavily on anticonvulsant drugs therapy. In an effort to elucidate the possible correlation between GDH and oxygen consumption and its possible participation in seizures mechanism here we report the effect of different anticonvulsant drugs on GDH activity and oxygen consumption in vitro as well as in vivo, studying oxidative deamination of GLU and reductive amination of $\alpha$-ketoglutarate $(\alpha \mathrm{K})$.

\section{Material and Methods}

2.1. Animals. Experiments were conducted in accordance with Helsinki Guide for the Care and Use of Laboratory Animals as adopted and promulgated by de EU Directive 2010/63/EU for animal experiments and approved by the institutional committee of ethics of E.N.C.B.

Albino male mice with a mean weight of $25 \mathrm{~g}$, fed ad libitum on a stock laboratory diet $(49.8 \%$ carbohydrates, $23.5 \%$ protein, $3.7 \%$ fat, minerals and added vitamins and amino acids; w/w), were used for the experiments. The animals were maintained on a $12 \mathrm{~h}$ light-dark cycle.

2.2. Reagents. Pyridoxal phosphate (PPAL) aminooxyacetic acid (AAOA), and GDH were purchased from Sigma Chemical co. (USA); Hydroxylamine (OHAMINE) was obtained from Merck (Dramstad, Germany). All anticonvulsants were dissolved in phosphate buffer $(\mathrm{pH}=7.4)$.

Standard analytical grade laboratory reagents were obtained from Merck or Sigma-Aldrich Chemical Corporation.

\subsection{GDH Activity Determination}

2.3.1. Tissue Processing. Animals without treatment (for in vitro assays) or after the treatment (for in vivo assays), were sacrificed and whole wet brains were removed. 25\% (wt/vol) homogenates was prepared with a Glas-Col tissue homogenizer in a $5 \%$ Triton X-100 solution. After centrifugation $(12,500 \mathrm{RPM} / 45 \mathrm{~min}$.), GDH activity was determined in the supernatant using a spectrophotometric assay according to the Strecker method [19]. Enzymatic activity assays were performed for the GLU oxidative deamination (forward reaction) and reductive amination of $\alpha \mathrm{K}$ (backward reaction).

2.3.2. Oxidative Deamination of Glutamate. Reaction medium was composed of phosphate buffer $0.05 \mathrm{M} \mathrm{pH}=7.6$, NAD $(0.036 \mathrm{M})$ and brains supernatant (see Section 2.3.1.). Reaction started by adding GLU $(0.5 \mathrm{M} \mathrm{pH}=7.0)$.

2.3.3. Reductive Amination of $\alpha$-Ketoglutarate. Reaction medium was composed of phosphate buffer $0.05 \mathrm{M} \mathrm{pH}=7.6$,
NADH $(0.0113 \mathrm{M}), \mathrm{NH}_{4} \mathrm{SO}_{4}(0.3 \mathrm{M})$, and brain supernatants (see Section 2.3.1.). Reaction started by addition of $\alpha$-ketoglutarate $(0.04 \mathrm{M} \mathrm{pH}=6.8)$.

\subsection{Effect of Anticonvulsant Drugs on Brain GDH Activity}

2.4.1. In Vitro Effect of Anticonvulsant Drugs on Brain GDH Activity. Different concentrations of PPAL, AAOA, and OHAMINE (between 0.001 and $0.00001 \mathrm{M}$ ) were added to the supernatant obtained from brain homogenates of animals. GDH activity was determined 10, 20, 30, and $60 \mathrm{~min}$ after reaction was initiated.

2.4.2. Effect of Aminooxyacetic Acid and Hydroxylamine on GDH of Different Sources. Effect of different concentrations of AAOA and OHAMINE (0.001, 0.0001, and 0.00001 M) on GDH activity of mouse brain, mouse liver (processed exactly as described for brains in Section 2.4.1.), and commercially available (from bovine liver, glycerol solution and ammonium-free diluted in phosphate buffer $0.05 \mathrm{M} \mathrm{pH}$ 7.6).

2.4.3. Effect of Different Concentrations of Ammonium on GDH Activity with and without Aminooxyacetic Acid and Hydroxylamine. GDH activity was determined using different concentrations of ammonium (between $40 \mu \mathrm{M}$ and $200 \mu \mathrm{M}$ ) with and without AAOA and OHAMINE 0.001 and $0.0001 \mathrm{M}$.

2.4.4. In Vivo Effect of Anticonvulsant Drugs on Brain GDH Activity. PPAL (50 mg/kg 30, 60 and $120 \mathrm{~min}$ ) OHAMINE $(40 \mathrm{mg} / \mathrm{kg} \mathrm{1}, 2$, and 4 hours), AAOA $(40 \mathrm{mg} / \mathrm{kg} \mathrm{30,60}$ and $120 \mathrm{~min}$ ), and HEPB (30, 60, and $120 \mathrm{~min}$ ) were administrated (IP) to groups of 5 animals at the dosage and during the time indicated. Animals were sacrificed and brains were quickly removed and processed for GDH activity determination as described in Section 2.3.

\subsection{Oxygen Consumption}

2.5.1. Tissue Processing. Animals were sacrificed, and once brains were excised, a $25 \%$ (wt/vol) homogenates in sucrose $0.25 \mathrm{M}$ was prepared. Homogenates were centrifuged at $3500 \mathrm{rpm}$ for 10 minutes, and supernatants were used to determine oxygen consumption by a polarographic method using a biological oxygen monitor YSI 5300. Determinations were performed using the same medium of reaction used for determination of oxidative deamination of GLU and reductive amination of $\alpha \mathrm{K}$ catalyzed by GDH (see Sections 2.3.2. and 2.3.3.).

2.5.2. In Vitro Effect of Anticonvulsant Drugs on Brain GDH Activity. To the supernatant obtained from animals without treatment as described above were added concentrations of $0.001,0.0001$, and $0.00001 \mathrm{M}$ of PPAL, AAOA, and OHAMINE and oxygen consumption was measured. 


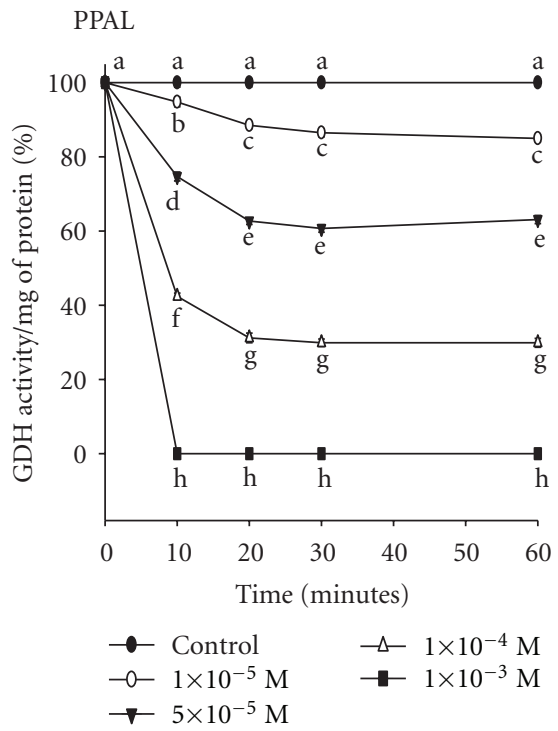

(a)

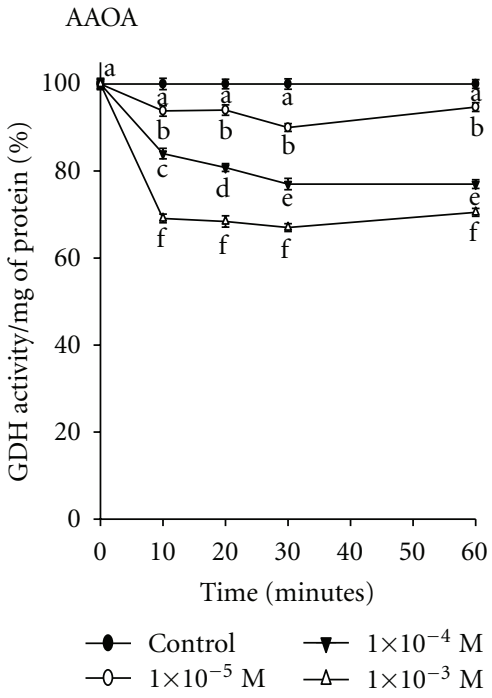

(b)

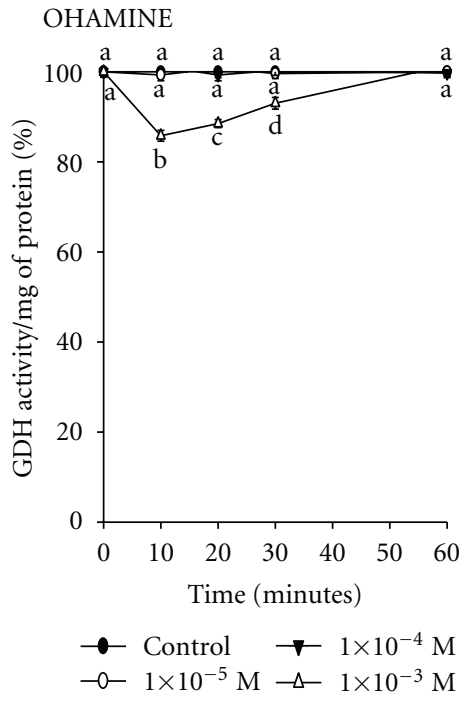

(c)

FIGURE 1: Effect in vitro of some anticonvulsant drugs on glutamate oxidative deamination catalized by mouse brain glutamate dehydrogenase. Glutamate dehydrogenase activity $(\mathrm{GDH})$ was determined in mouse brain homogenates $(1: 5 \mathrm{w}: \mathrm{v}$ on phosphates $0.05 \mathrm{M} \mathrm{pH}=7.6$ buffer) with different concentrations of pyridoxal phosphate (PPAL), hydroxylamine (OHAMINE) and aminooxyacetic acid (AAOA) with glutamic acid as substrate, using a spectrophotometric method (see Section 2$)$. Results are means \pm SEM $(n>4)$. ANOVA test was applied and different characters were used to indicate statistically different groups as compared with the control $(P>0.01$, Tuckey's test).

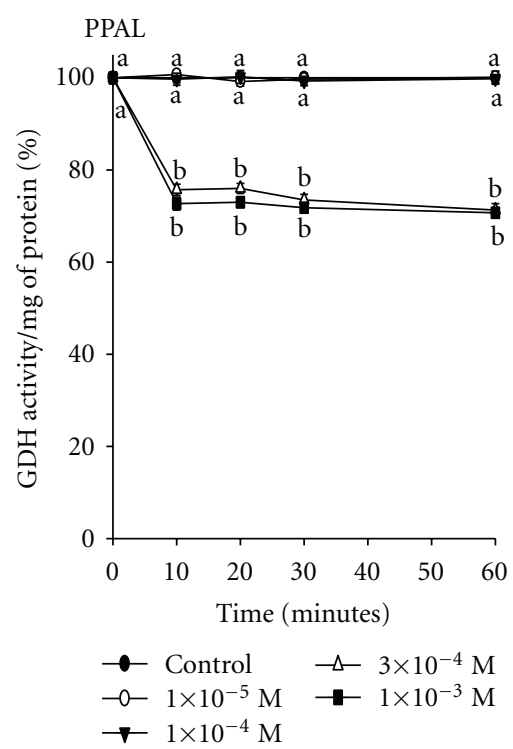

(a)
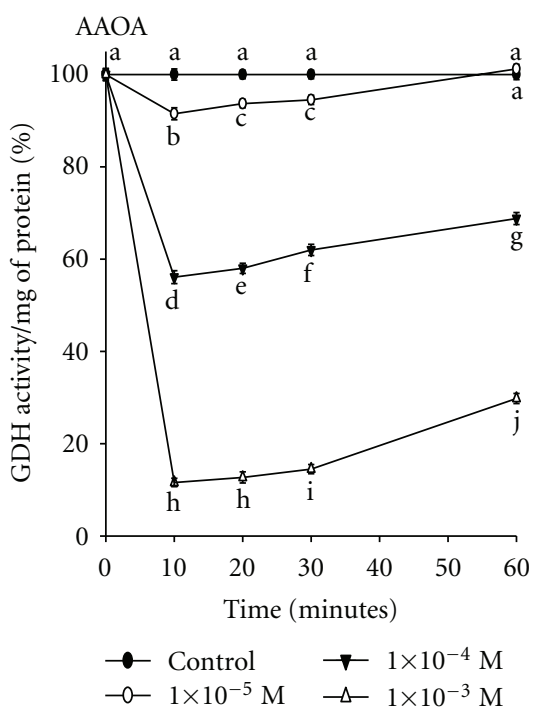

(b)

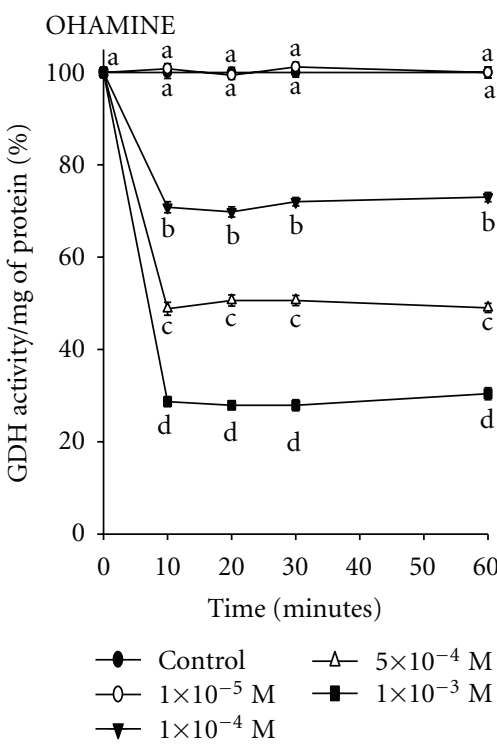

(c)

FIGURE 2: Effect in vitro of some anticonvulsant drugs on reductive amination of $\alpha$-ketoglutarate catalaized by mouse brain glutamate dehydrogenase. Glutamate dehydrogenase activity (GDH) was determined in mouse brain homogenates ( $1: 5 \mathrm{w}: \mathrm{v}$ on phosphates $0.05 \mathrm{M}$ $\mathrm{pH}=7.6$ buffer) with different concentrations of pyridoxal phosphate (PPAL), hydroxylamine (OHAMINE), and aminooxyacetic acid (AAOA) with $\alpha$-ketoglutarate as substrate, using a spectrophotometric method (see Section 2 ). Results are means \pm SEM ( $n>4$ ). ANOVA test was applied and different characters were used to indicate statistically different groups as compared with the control $(P>0.01$, Tuckey's test). 


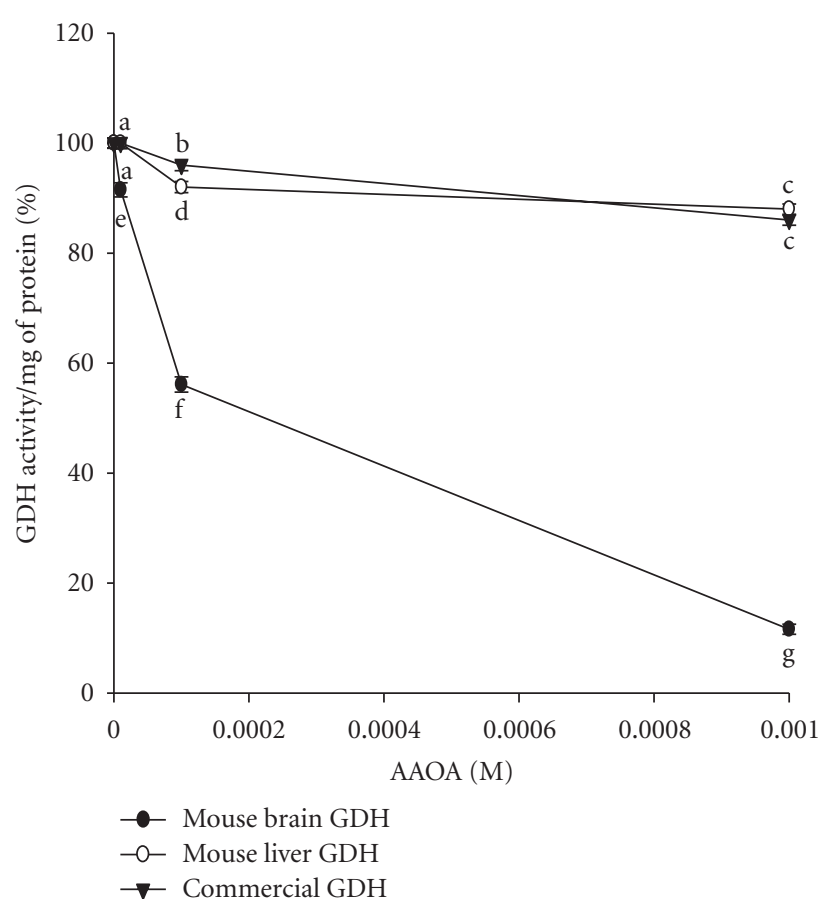

(a)

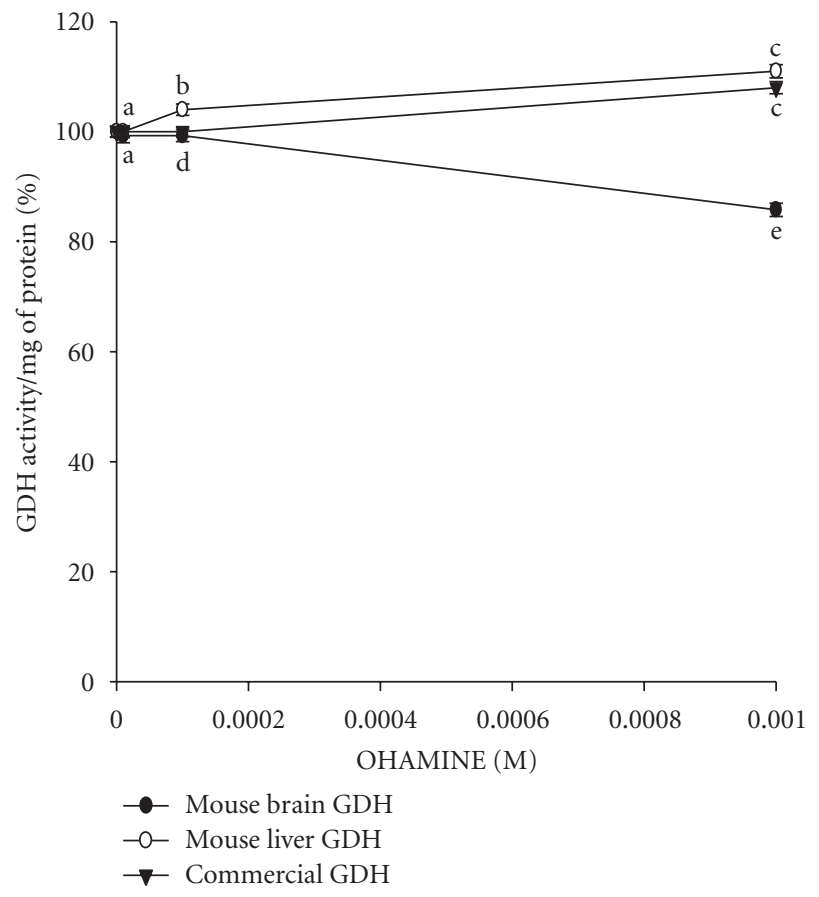

(b)

FIGURE 3: Effect in vitro of aminooxiacetic and hydroxylamine on glutamate dehydrogenase activity of different sources. Effect of different concentrations of aminooxyacetic acid (AAOA), and hydroxylamine (OHAMINE) on reductive amination of $\alpha$-ketoglutarate and oxidative deamination of glutamate respectively, catalyzed by glutamate dehydrogenase commercially available (from bovine liver), mouse brain, and liver homogenates ( $1: 5 \mathrm{w}: \mathrm{v}$ on phosphates $0.05 \mathrm{M} \mathrm{pH}=7.6$ buffer), using a spectrophotometric method (see Section 2). Results are means \pm SEM $(n>4)$. ANOVA test was applied, and different characters were used to indicate statistically different groups as compared with the control $(P>0.01$, Tuckey's test $)$.

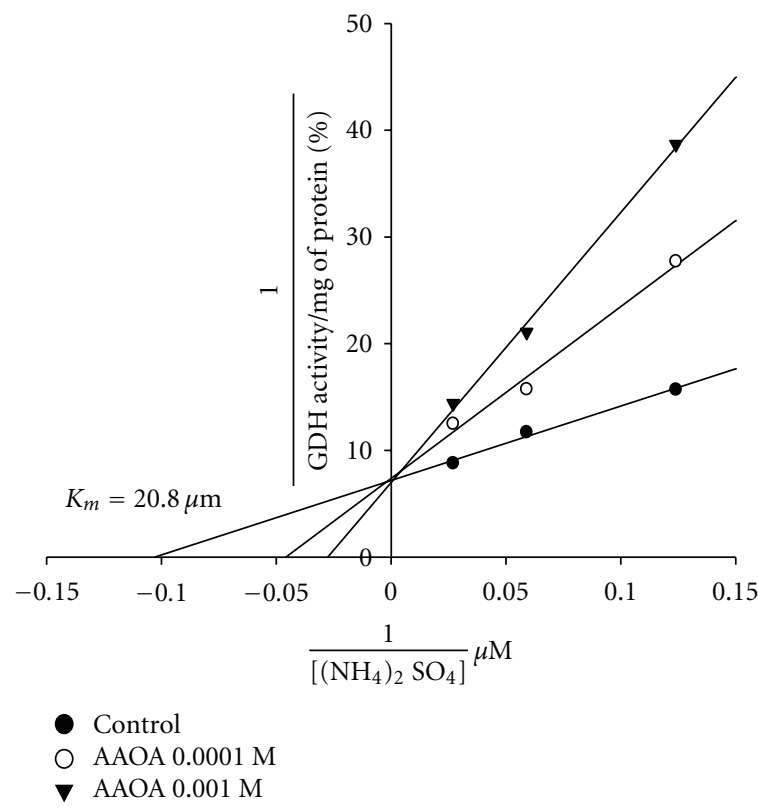

(a)

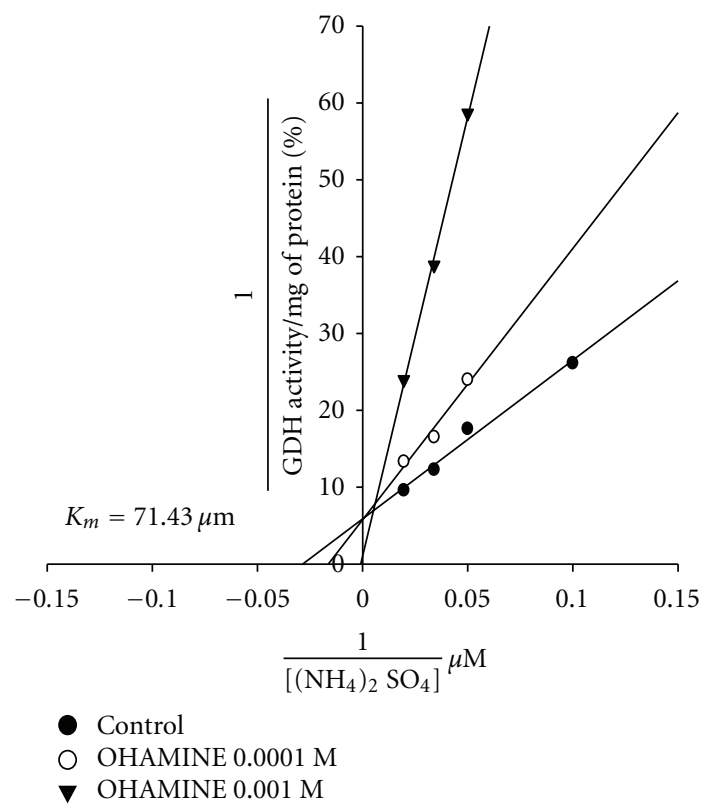

(b)

FIGURE 4: Effect of ammonium sulphate on the inhibitory activity of aminooxyacetic acid and hydroxylamine on mouse brain glutamate dehydrogenase. Glutamate dehydrogenase activity (GDH) was determined in mouse brain homogenates ( $1: 5 \mathrm{w}: \mathrm{v}$ on phosphates $0.05 \mathrm{M}$ $\mathrm{pH}=7.6$ buffer) for reductive amination of $\alpha$-ketoglutarate employing different concentrations of ammonium sulphate, with and without aminooxyacetic acid (AAOA, (a)) and hydroxylamine (OHAMINE, (b)), using a spectrophotometric method (see Section 2). Reciprocal of GDH activity are plotted against reciprocal of ammonium concentration. 


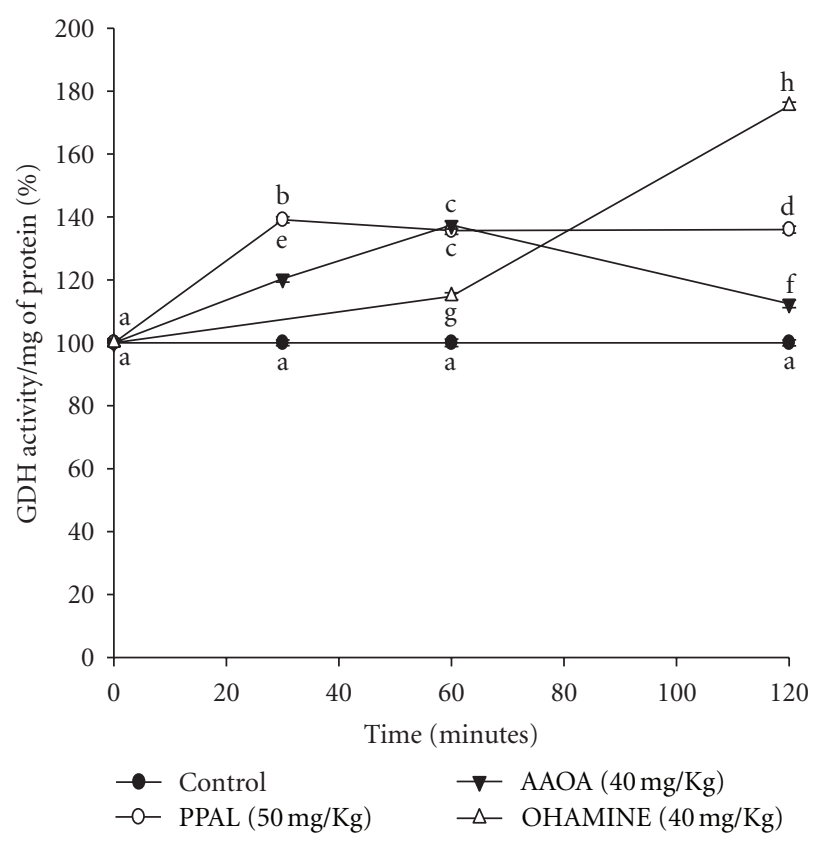

(a) Glutamate

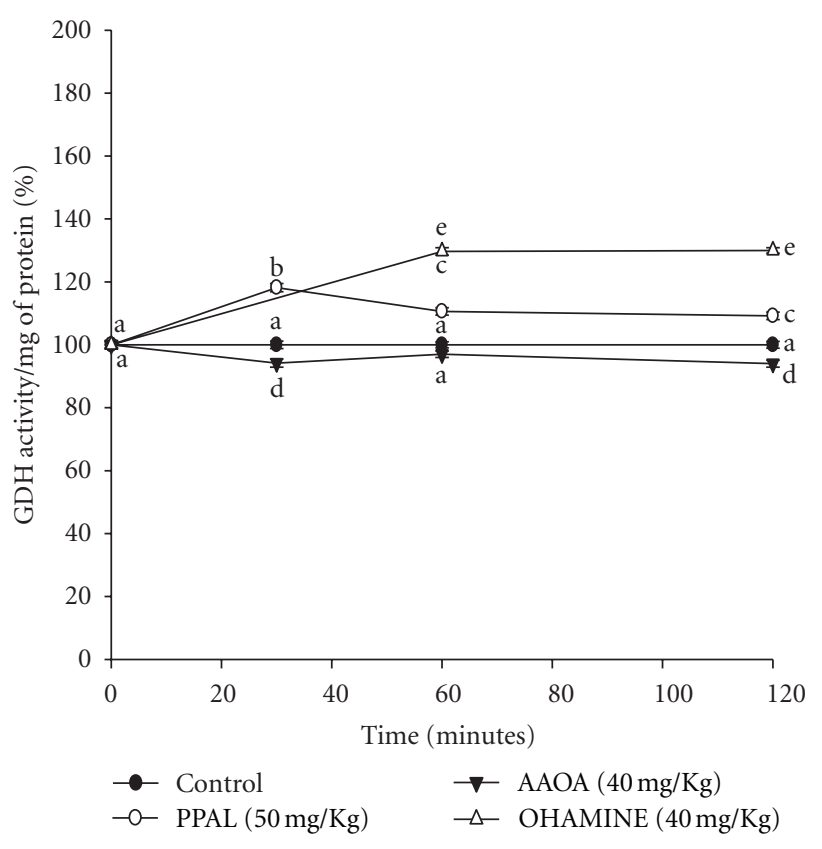

(b) $\alpha$-ketoglutarate

FIGURE 5: Effect in vivo of some anticonvulsant drugs on mouse brain glutamate dehydrogenase. Animals received pyridoxal phosphate (PPAL, $50 \mathrm{mg} / \mathrm{Kg}$ ), hydroxylamine (OHAMINE, $40 \mathrm{mg} / \mathrm{kg}$ ), and aminooxyacetic acid (AAOA, $40 \mathrm{mg} / \mathrm{Kg}$ ). After different times of treatment, glutamate dehydrogenase activity $(\mathrm{GDH})$ was determined in brain homogenates $(1: 5 \mathrm{w}: \mathrm{v}$ on phosphates $0.05 \mathrm{M} \mathrm{pH}=7.6$ buffer $)$ for oxidative deamination of glutamate and reductive amination of $\alpha$-ketoglutarate, using a spectrophotometric method (see Section 2). Results are means \pm SEM $(n>4)$. ANOVA test was applied and different characters were used to indicate statistically different groups as compared with the control $(P>0.01$, Tuckey's test $)$.

2.5.3. In Vivo Effect of Anticonvulsant Drugs on Brain GDH Activity. Groups of five animals received PPAL $(50 \mathrm{mg} / \mathrm{kg})$, AAOA $(40 \mathrm{mg} / \mathrm{kg})$ and OHAMINE $(40 \mathrm{mg} / \mathrm{kg})$ IP and after 1 hour, animals were sacrificed and brains were quickly removed and once processed oxygen consumption determination was performed.

2.6. Protein Levels. Protein concentration was determined in supernatants by Lowry method [20].

2.7. Statistical Analyses. All results that were normalized against control were in turn mean $\pm \mathrm{SE}$ values of at least four determinations $(n \geq 4)$. GDH activity was compared between groups by using one-way analysis of variance (ANOVA), followed by Tukey's multiple comparisons. A $P$ value of 0.01 was regarded as statistically significant.

\section{Results}

3.1. Anticonvulsant Drugs and Brain GDH Activity In Vitro. Figures 1 and 2 represent the effect of anticonvulsants on GDH activity in vitro. Results show that PPAL decreased GLU oxidative deamination in a concentration dependent manner, it was totally inhibited at $0.001 \mathrm{M}$.

AAOA and OHAMINE decreased GDH activity using GLU as substrate 30 and $18 \%$, respectively. Although this inhibition is significant, the effect produced by these substances is higher in reductive amination of $\alpha \mathrm{K}$, reaching 90 and $70 \%$ of inhibition, respectively. PPAL inhibited reductive amination of $\alpha \mathrm{K}$, but only $25 \%$ as maximum.

\subsection{Effect of Aminooxyacetic Acid and Hydroxylamine on} GDH of Different Sources. A comparison of effects of AAOA and OHAMINE on GDH from different sources is shown in Figure 3, where can be appreciated that whereas mouse liver and commercial GDH inhibited only slightly reductive amination of $\alpha \mathrm{K}$, mouse brain GDH activity is decreased about $70 \%$.

3.3. Effect of Ammonium on GDH Activity with and without Aminooxyacetic Acid and Hydroxylamine. Lineweaver-Burk graph for AAOA and OHAMINE effects on GDH activity, using ammonium as substrate, can be observed in Figure 4 . Results indicate a competitive inhibition of brain GDH activity for both anticonvulsants, with a Km value of $20.8 \mu \mathrm{M}$, and $71.43 \mu \mathrm{M}$, respectively.

3.4. Anticonvulsant Drugs and GDH Activity In Vivo. The effect of administration of anticonvulsant drugs on brain GDH activity was also investigated. In vivo results are different to the observed in vitro, since an increase in GLU oxidative deamination catalyzed by GDH was found in all cases (Figure 5(a)). Anticonvulsant drugs caused also an increase on $\alpha$ ketoglutaric reductive amination (except for AAOA, Figure 5(b)), but this effect was of smaller magnitude compared with the observed for GLU utilization. 


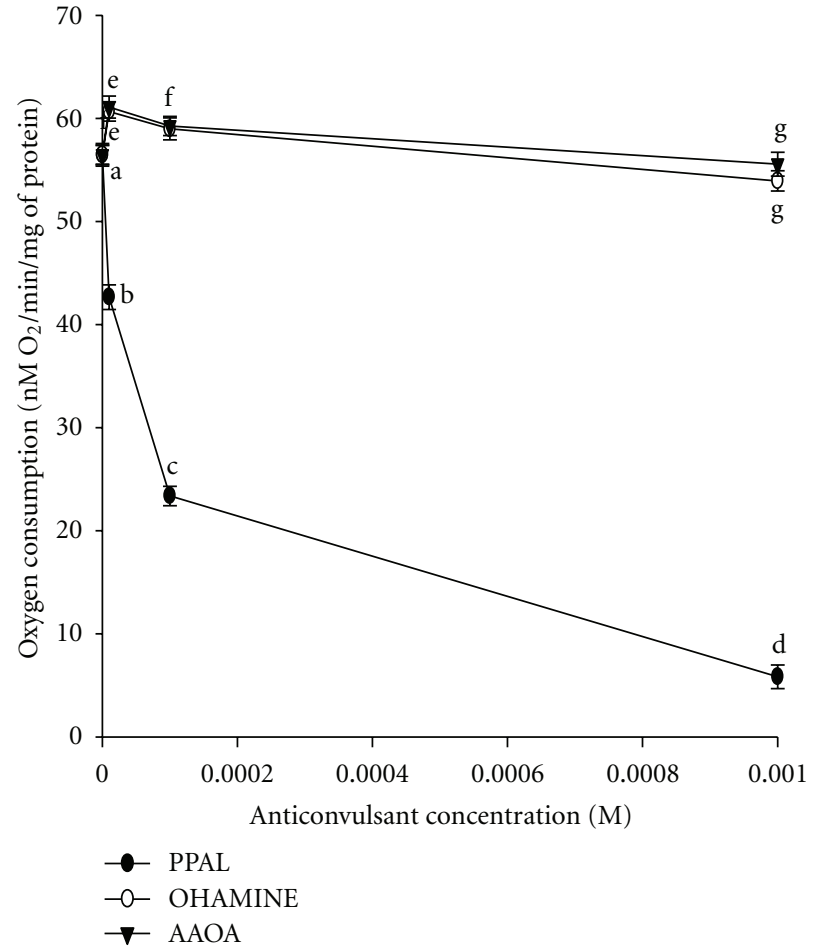

(a) Glutamate

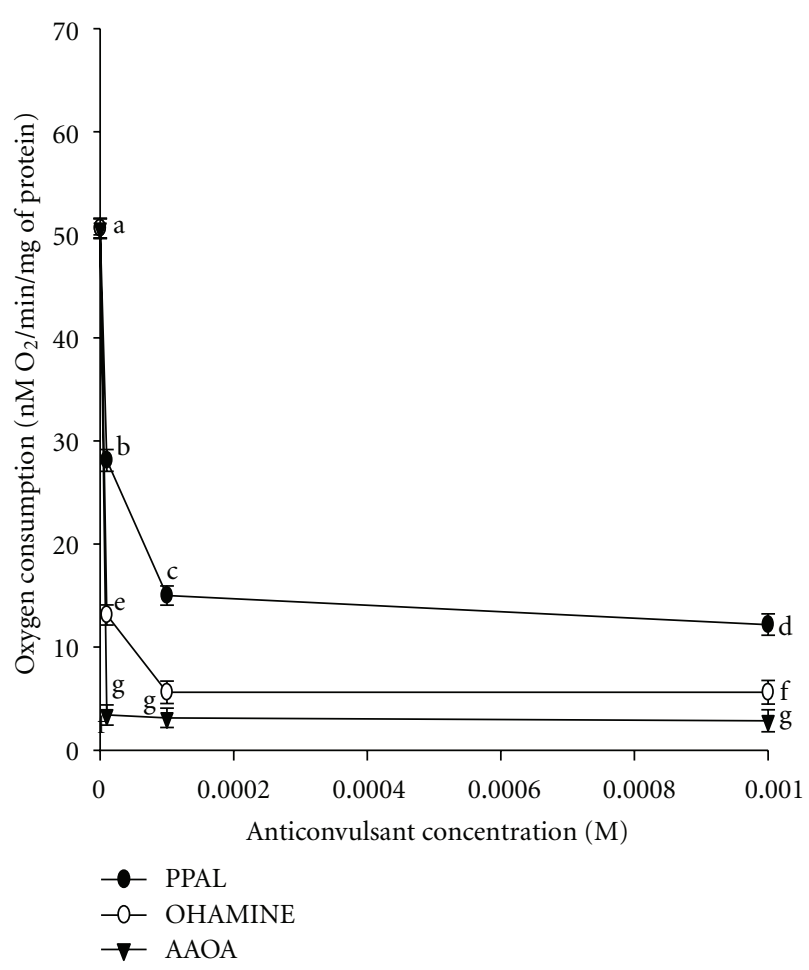

(b) $\alpha$-ketoglutarate

FIGURE 6: Effect in vitro of some anticonvuulsant drugs on oxygen consumption. Different concentrations of pyridoxal phosphate (PPAL), hydroxylamine (OHAMINE), and aminooxyacetic acid (AAOA) were added and oxygen consumption was determined in mouse brain homogenates $(25 \% \mathrm{wt} / \mathrm{vol}$ in sucrose $0.25 \mathrm{M}$ ) by a polarographic method using glutamate (a) and $\alpha$-ketoglutarate (b) as substrates (see Section 2). Results are means \pm SEM $(n>4)$. ANOVA test was applied and different characters were used to indicate statistically different groups as compared with the control $(P>0.01$, Tuckey's test $)$.

3.5. Anticonvulsant Drugs and Oxygen Consumption In Vitro. As observed in GDH activity, in vitro effects of anticonvulsant drugs on oxygen consumption depend on type of substrate. Results (Figure 6) show that PPAL decreased oxygen consumption when GLU was the substrate. AAOA and OHAMINE decrease oxygen consumption with $\alpha$ ketoglutaric acid as substrate.

3.6. Anticonvulsant Drugs and Oxygen Consumption In Vivo. While anticonvulsants had different in vitro effects, in vivo all of them decreased oxygen consumption, but this happened only when GLU was the substrate (Figure 7(a)). With $\alpha$ ketoglutaric acid, we found decreases (with PPAL and HAMINE) as well as increases (AAOA) in oxygen consumption (Figure 7(b)).

\section{Discussion}

Anticonvulsants affected reductive amination of $\alpha \mathrm{K}$ and/or oxidative deamination of GLU in different degree, so the results of this work show the relevance of considering not only one but both directions of GDH reaction.

Results analyzing inhibition of oxidative deamination of GLU induced by PPAL in vitro are in concordance with other reports $[21,22]$. In contrast, decreased reductive amination of $\alpha \mathrm{K}$ caused by AAOA and OHAMINE is in opposition with other authors, that report no effect of these drugs on GDH activity using either GLU or $\alpha \mathrm{K}$ as substrate [23]. Although some studies indicate GDH isolated from bovine liver and brain have similar kinetic and allosteric properties $[24,25]$, kinetic differences have been demonstrated between the liver and brain GDH in the rat $[26,27]$. Comparison of effects on commercially available (bovine liver), liver and mouse brain GDH activity for both AAOA and OHAMINE, suggest that the reason of this apparent discrepancy is the enzyme source. For mouse brain GDH, inhibition of $\alpha \mathrm{K}$ reductive amination in vitro by AAOA and OHAMINE is competitive with ammonium, which could be explained by their structure resembling this group in both cases.

Since AAOA increases GABA levels reaching a maximal at 6 hours, but protection against electroshock and pentylenetetrazole were maximal $1 \mathrm{hr}$ after administration and had vanished after 6 hours [28], it is considered that its anticonvulsant action has a dual mechanism. The first one, involving GABA metabolism, most effective $6 \mathrm{~h}$ after administration, and the other, not involving GABA, maximally effective $1.5 \mathrm{~h}$ after AAOA injection [29], in which is proposed that GDH may participate regulating GLU levels.

Contrarily to the results observed in vitro, anticonvulsant in vivo produced a significant increase of GLU oxidative 


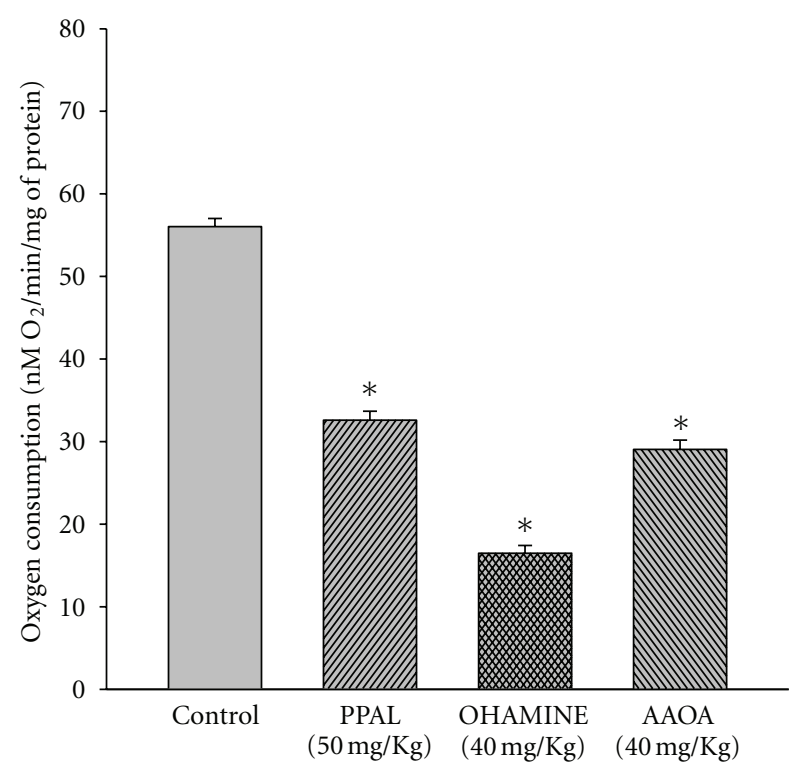

(a) Glutamate

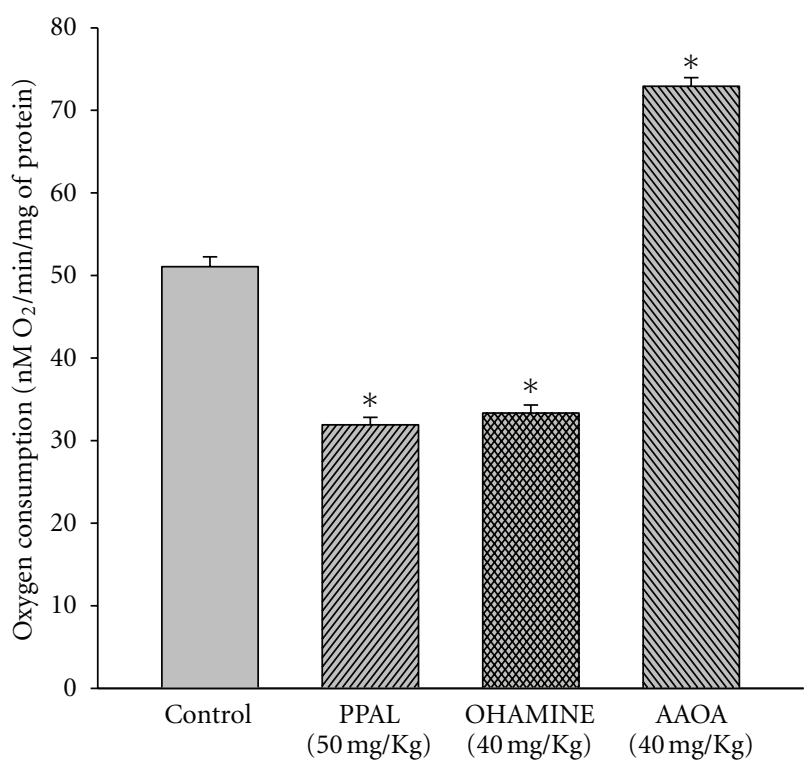

(b) $\alpha$-ketoglutarate
The differences between in vitro and in vivo effects of these drugs could be attributed to the change on brain energy requirements, since GDH activity is highly regulated by ATP, ADP, GTP, GDP, and other metabolites which indicate energetic needs. In other words, in vitro results are not under metabolic regulation as they are in vivo. This hypothesis seems to be supported by the results obtained analyzing the effects of anticonvulsants on oxygen consumption, which indicate different metabolic activity, thus different energetic requirements, in vivo as compared with activity in vitro.

Figure 6 shows that, in vitro, anticonvulsant tested diminished oxygen consumption, but it happened just when the same substrate in which these drugs decreased GDH activity was used. On the other hand, in vivo results indicate that anticonvulsants not only increased oxidative deamination of GLU but also decreased oxygen consumption with this substrate. Hence, it could be proposed that changes in GDH activity have a repercussion on oxygen consumption, but although in vivo $\alpha \mathrm{K}$ is generated by brain GDH, it is not oxidized in Krebs cycle, maybe because brain metabolism is decreased and does not require additional energy.

Altogether, our results showed that anticonvulsant effect of tested substances could involve a decrease in GLU levels by GDH activation, avoiding its extracellular accumulation and toxicity. Besides, changes in GDH activity have repercussions on oxygen consumption, which indicates that the enzyme activity is highly regulated by energy requirements for metabolic activity. Then, GDH may participate in neuronal excitability regulation and may represent a key enzyme in seizures mechanism.

\section{Conclusions}

GDH may participate in regulation of GLU and indirectly GABA levels, hence in neuronal excitability. There is a relation between oxygen consumption and GDH activity, which indicate that the enzyme activity is highly regulated by energy requirements for metabolic activity. Results reinforce the important role of GDH in GLU metabolism [30], and suggest that may represent a key enzyme in seizures mechanism.

\section{Acknowledgment}

FIGURE 7: Effect in vivo of some anticonvulsant drugs on oxy-
gen consumption. Animals received pyridoxal phosphate (PPAL, $50 \mathrm{mg} / \mathrm{Kg}$ ), hydroxylamine (OHAMINE, $40 \mathrm{mg} / \mathrm{kg}$ ), and aminooxyacetic acid (AAOA, $40 \mathrm{mg} / \mathrm{Kg}$ ). After the treatment, oxygen consumption was determined in brain homogenates $(25 \% \mathrm{wt} / \mathrm{vol}$ in sucrose $0.25 \mathrm{M}$ ), by a polarographic method using glutamate (a) and $\alpha$-ketoglutarate (b) as substrates (see Section 2). Results are means \pm SEM $(n>4)$. ${ }^{*}$ Statistically significant differences as compared with the control $(P>0.01$, Student's $t$ test).

deamination, which would lead to a decrease in GLU levels, hence a diminution of its excitatory effect. The increase and only slight inhibition in $\alpha \mathrm{K}$ reductive amination produced by OHAMINE and AAOA, respectively, are also opposite to their effects in vitro.
This work was supported by Section of Postgraduate Studies and Research, Academic Secretary of National Polytechnic Institute, México.

\section{References}

[1] H. S. White, "Comparative anticonvulsant and mechanistic profile of the established and newer antiepileptic drugs," Epilepsia, vol. 40, supplement 1, pp. S2-S10, 1999.

[2] E. M. Urbanska, S. J. Czuczwar, Z. Kleinrok, and W. A. Turski, "Excitatory amino acids in epilepsy," Restorative Neurology and Neuroscience, vol. 13, no. 1-2, pp. 25-39, 1998.

[3] J. Doherty and R. Dingledine, "The roles of metabotropic glutamate receptors in seizures and epilepsy," Current Drug Targets-CNS \& Neurological Disorders, vol. 1, no. 3, pp. 251260, 2002. 
[4] R. Dingledine, K. Borges, D. Bowie, and S. F. Traynelis, "The glutamate receptor ion channels," Pharmacological Reviews, vol. 51, no. 1, pp. 7-61, 1999.

[5] W. Löscher, "New visions in the pharmacology of anticonvulsion," European Journal of Pharmacology, vol. 342, no. 1, pp. $1-13,1998$.

[6] C. G. Parsons, W. Danysz, and G. Quack, "Glutamate in CNS disorders as a target for drug development: an update," Drug News and Perspectives, vol. 11, no. 9, pp. 523-569, 1998.

[7] D. E. Pellegrini-Giampietro, J. A. Gorter, M. V. L. Bennett, and R. S. Zukin, "The GluR2 (GluR-B) hypothesis: $\mathrm{Ca}^{2+}$ permeable AMPA receptors in neurological disorders," Trends in Neurosciences, vol. 20, no. 10, pp. 464-470, 1997.

[8] A. Plaitakis, P. Flessas, A. B. Natsiou, and P. Shashidharan, "Glutamate dehydrogenase deficiency in cerebellar degenerations: clinical, biochemical and molecular genetic aspects," Canadian Journal of Neurological Sciences, vol. 20, supplement 3, pp. S109-S116, 1993.

[9] G. H. Malthankar-Phatak, N. De Lanerolle, T. Eid et al., "Differential glutamate dehydrogenase (GDH) activity profile in patients with temporal lobe epilepsy," Epilepsia, vol. 47, no. 8, pp. 1292-1299, 2006.

[10] A. L. Sherwin, "Neuroactive amino acids in focally epileptic human brain: a review," Neurochemical Research, vol. 24, no. 11, pp. 1387-1395, 1999.

[11] D. M. Raizen, A. Brooks-Kayal, L. Steinkrauss, G. I. Tennekoon, C. A. Stanley, and A. Kelly, "Central nervous system hyperexcitability associated with glutamate dehydrogenase gain of function mutations," Journal of Pediatrics, vol. 146, no. 3, pp. 388-394, 2005.

[12] F. Cerchia and P. Mantegazzini, "Convulsive action of high pressure oxygen in various animal species," Annali di Medicina Navale, vol. 61, no. 2, pp. 127-136, 1956.

[13] J. D. Wood, W. J. Watson, and A. J. Ducker, "Oxygen poisoning in various mammalian species and the possible role of gammaaminobutyric acid metabolism," Journal of Neurochemistry, vol. 14, no. 11, pp. 1067-1074, 1967.

[14] K. Gulati, A. Ray, G. Pal, and V. K. Vijayan, "Possible role of free radicals in theophylline-induced seizures in mice," Pharmacology Biochemistry and Behavior, vol. 82, no. 1, pp. 241-245, 2005.

[15] M. Prigol, E. A. Wilhelm, C. C. Schneider, J. B. T. Rocha, C. W. Nogueira, and G. Zeni, "Involvement of oxidative stress in seizures induced by diphenyl diselenide in rat pups," Brain Research, vol. 1147, no. 1, pp. 226-232, 2007.

[16] S. Fujimoto, H. Katsuki, T. Kume, S. Kaneko, and A. Akaike, "Mechanisms of oxygen glucose deprivation-induced glutamate release from cerebrocortical slice cultures," Neuroscience Research, vol. 50, no. 2, pp. 179-187, 2004.

[17] L. L. Guyot, F. G. Diaz, M. H. O’Regan, S. McLeod, H. Park, and J. W. Phillis, "Real-time measurement of glutamate release from the ischemic penumbra of the rat cerebral cortex using a focal middle cerebral artery occlusion model," Neuroscience Letters, vol. 299, no. 1-2, pp. 37-40, 2001.

[18] A. Melani, L. Pantoni, C. Corsi et al., "Striatal outflow of adenosine, excitatory amino acids, $\gamma$-aminobutyric acid, and taurine in awake freely moving rats after middle cerebral artery occlusion: correlations with neurological deficit and histopathological damage," Stroke, vol. 30, no. 11, pp. 2448 2455, 1999.

[19] H. J. Strecker, "Glutamic dehydrogenase," Archives of Biochemistry and Biophysics, vol. 46, no. 1, pp. 128-140, 1953.

[20] O. H. Lowry, N. J. Rosebrough, A. L. Farr, and R. J. Randall, "Protein measurement with the Folin phenol reagent," The
Journal of Biological Chemistry, vol. 193, no. 1, pp. 265-275, 1951.

[21] B. M. Anderson, C. D. Anderson, and J. E. Churchich, "Inhibition of glutamic dehydrogenase by pyridoxal $5^{\prime}$-phosphate," Biochemistry, vol. 5, no. 9, pp. 2893-2900, 1966.

[22] J. Torreilles, J. Marchand, and M. C. Guerin, "NAD(P) adducts as protective agents against glutamate dehydrogenase inactivation by pyridoxal $5^{\prime}$-phosphate: a tool for the study of oxidized coenzyme activated state in enzymatic evolutive and abortive complexes," Biochimie, vol. 64, no. 6, pp. 429-434, 1982.

[23] E. Kun and B. Achmatowicz, "Studies with specific enzyme inhibitors. Ix. Selective inhibitory effects of substrate analogues on the catalytic activity of crystalline glutamate dehydrogenase," The Journal of Biological Chemistry, vol. 240, pp. 2619-2627, 1965.

[24] N. Talal and G. M. Tomkins, "Allosteric properties of glutamate dehydrogenases from different sources," Science, vol. 146, no. 3649, pp. 1309-1311, 1964.

[25] A. D. McCarthy, J. M. Walker, and K. F. Tipton, "Purification of glutamate dehydrogenase from ox brain and liver. Evidence that commercially available preparations of the enzyme from ox liver have suffered proteolytic cleavage," Biochemical Journal, vol. 191, no. 2, pp. 605-611, 1980.

[26] P. Y. Chee, J. L. Dahl, and L. A. Fahien, "The purification and properties of rat brain glutamate dehydrogenase," Journal of Neurochemistry, vol. 33, no. 1, pp. 53-60, 1979.

[27] V. G. Erwin, "Enhancement of brain glutamate dehydrogenase activity and glutamate oxidation by adenine nucleotides," Molecular Pharmacology, vol. 5, no. 6, pp. 615-624, 1969.

[28] W. Loscher and M. Vetter, "In vivo effects of aminooxyacetic acid and valproic acid on nerve terminal (synaptosomal) GABA levels in discrete brain areas of the rat. Correlation to pharmacological activities," Biochemical Pharmacology, vol. 34, no. 10, pp. 1747-1756, 1985.

[29] J. D. Wood and S. J. Peekser, "A dual mechanism for the anticonvulsant action of aminooxyacetic acid," Canadian Journal of Physiology and Pharmacology, vol. 54, no. 4, pp. 534540, 1976.

[30] J. W. Geddes and J. D. Wood, "Changes in the amino acid content of nerve endings (synaptosomes) induced by drugs that alter the metabolism of glutamate and $\gamma$-aminobutyric acid," Journal of Neurochemistry, vol. 42, no. 1, pp. 16-24, 1984. 

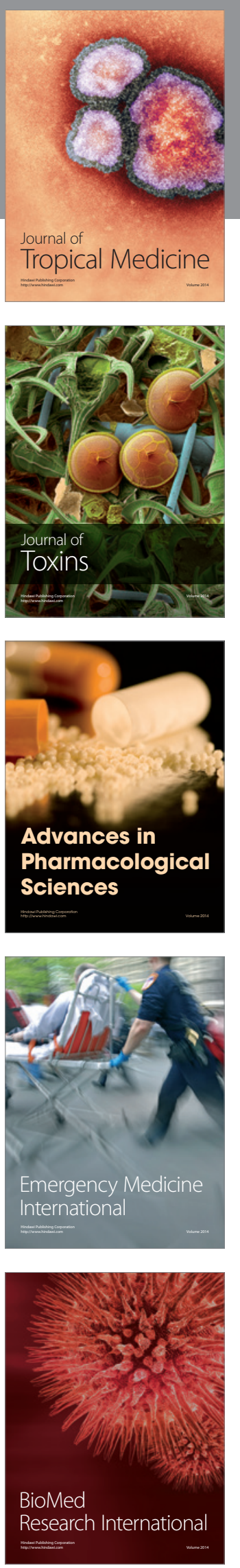
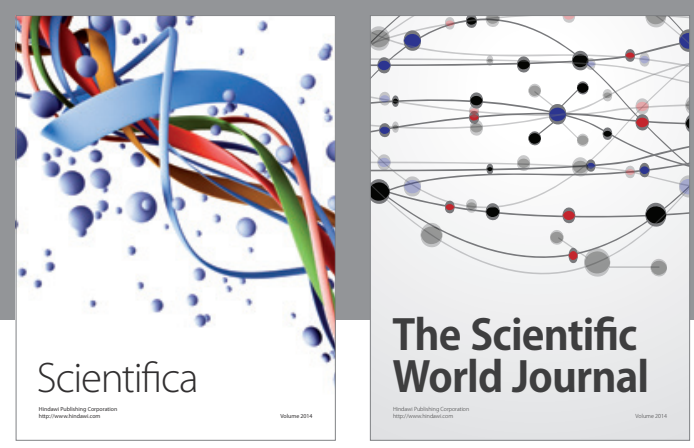

The Scientific World Journal
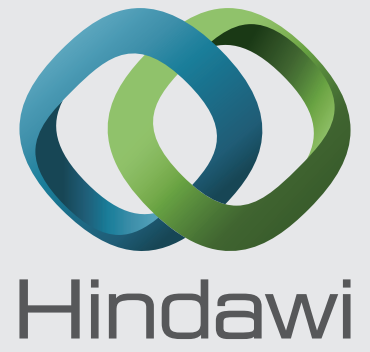

Submit your manuscripts at

http://www.hindawi.com
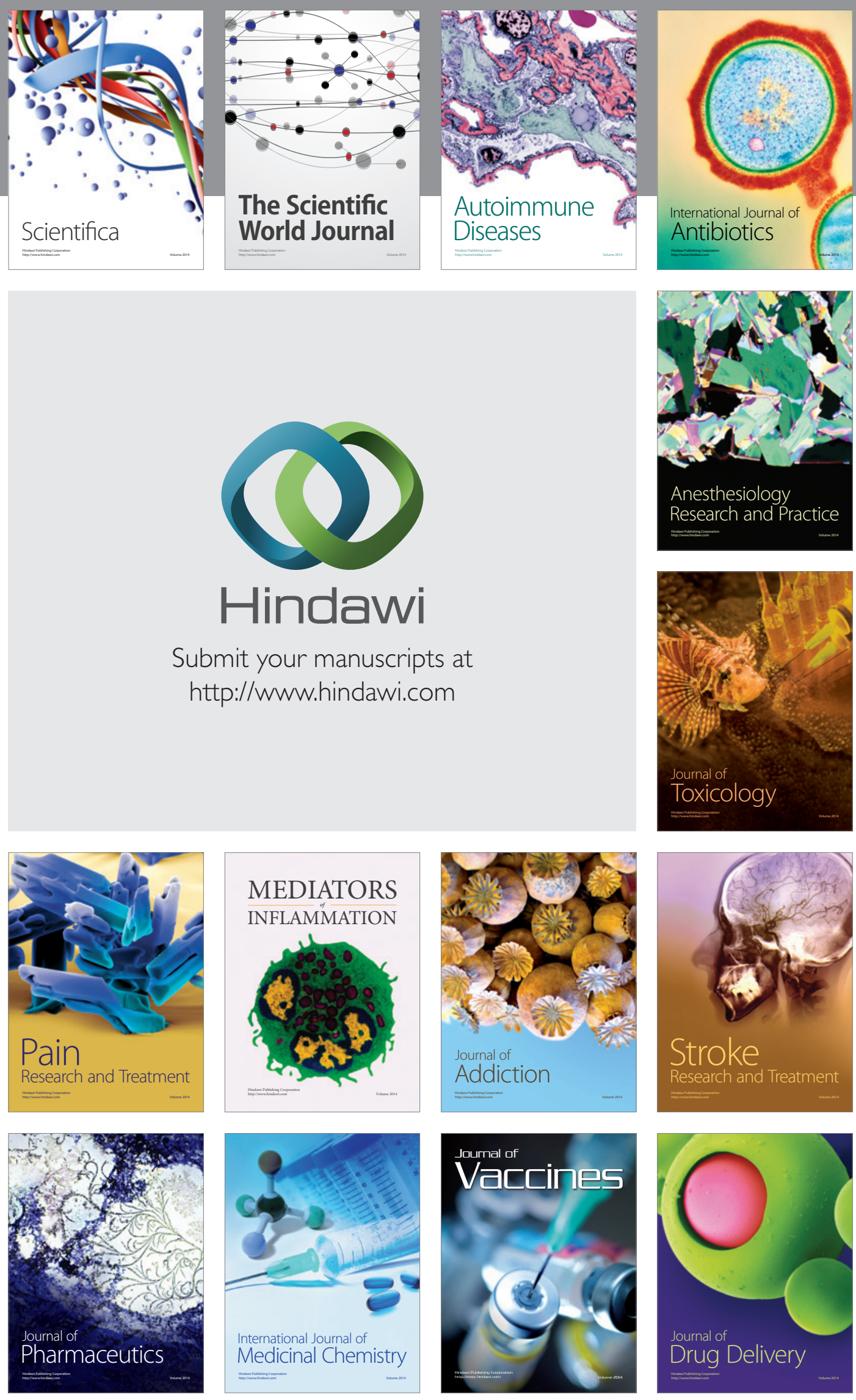